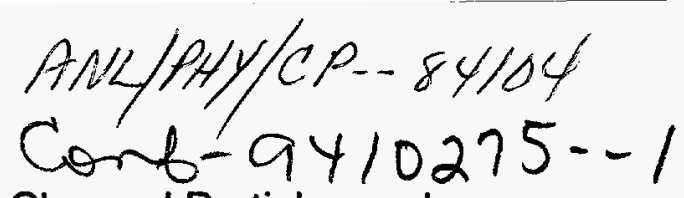

Invited paper for the Symposium on "The Interaction of Charged Particles and

Radiation with Matter" in honor of R.H. Ritchie on his 70th birthday.

Oak Ridge, Tennessee, October 23- 25, 1994

(To be published in Nucl. Instr. Meths.)

\title{
The Interactions of High-Energy, Highly Charged Xe Ions with Buckyballs
}

R. Ali, H.G. Berry, S. Cheng, ${ }^{*}$ R.W. Dunford, H. Esbensen, D.S. Gemmell, E.P. Kanter, T. LeBrun, and L. Young, Physics Division, Argonne National Laboratory, Argonne, IL 60439, and W. Bauer, NSCL, Michigan State University, East Lansing, MI 48824, USA.

Abstract: Ionization and fragmentation have been measured for $\mathrm{C}_{60}$ molecules bombarded by highly charged (up to 35+) xenon ions with energies ranging up to 625 $\mathrm{MeV}$. The observed mass distribution of positively charged fragments is explained in terms of a theoretical model indicating that the total interaction cross section contains roughly equal contributions from a) excitation of the giant plasmon resonance, and b) large-energy-transfer processes that lead to multiple fragmentation of the molecule. Preliminary results of measurements on VUV photons emitted in these interactions are also presented.

The relatively recent discovery ${ }^{1}$ of the highly stable and symmetric quasi-spherical molecule $C_{60}$ and related fullerenes has led to intense studies on a wide variety of the properties of this new allotropic form of carbon. Research on these open cage-like structures ("buckyballs") received a big boost when methods were developed ${ }^{2}$ to produce them in macroscopic quantities.

An interesting aspect of the fullerenes is that they represent intermediate structures lying between the solid-state and the atomic scales. As is observed in the solid-state and in atomic (and indeed nuclear) systems, fullerenes also exhibit collective oscillations. This property is of fundamental importance in the measurements we report here and provides a direct link to this Symposium in honor of Rufus Ritchie who has contributed so much over the years to the development of the understanding of collective modes.

Among many approaches to the study of fullerenes, the use of atomic collision techniques offers a powerful tool for investigating their structures and their dynamics. Indeed several collision studies have already been reported ${ }^{3}$. Our experiments were performed at Argonne's 


\section{DISCLAIMER}

Portions of this document may be illegible in electronic image products. Images are produced from the best available original document. 
ATLAS heavy-ion linear accelerator, where we studied the interactions of high-energy (up to 625 $\mathrm{MeV}$ ), highly charged (up to 35+) Xe ions with $C_{60}$. These beam energies exceeded those used in previous work by several orders of magnitude. The high values of projectile velocity and charge state result in excitation and decay processes differing significantly from those seen in studies at lower energies (see, for example, Ref. 4).

Our $C_{60}$ vapor target was formed from $99.5 \%$ pure material heated in àn oven to $475^{\circ} \mathrm{C}$. A time-of-flight (TOF) spectrometer system (see Fig. 1) was located at $90^{\circ}$ to the incident beam. Grids around the target region were biased with voltages to extract positively charged fragments and to inject them into a $20-\mathrm{cm}-$ long gridded flight tube and thence into a micro-channel-plate detector. A "beam sweeper" at ATLAS was employed so as to allow one 0.4-ns-wide beam pulse to reach the target every $10 \mu \mathrm{s}$. TOF spectra were obtained using a "multi-hit" time digitizer with the "start" signal coming from the detector and the "stop" signal from the accelerator's timing system.

Fig. 2 shows the TOF spectrum and its equivalent calibration in terms of M/Q, the ratio of fragment mass to charge. The peaks in Fig. 2 that correspond to interactions of the projectiles with $C_{60}$ fall into three categories :

1) Peaks due to singly, doubly, triply, and (possibly) quadruply ionized $C_{60}$.

2) Peaks corresponding to the losses of even-numbered neutral carbon fragments.

3) Peaks corresponding to the sequence of singly charged fragments $C_{n}^{+}$, with $n$ assuming all values from 1 to at least 19 . These peaks alternate in intensity up to around $n=9$ with the oddnumbered peaks being more intense than the even-numbered. Above $n=9$, the most intense peaks appear to be $n=11,15$, and probably 19 . The intensity variations mirror those seen in other studies and indicate the relative stabilities of linear chain $(n \leq 9)$ and cyclic $(n \gtrsim 9)$ structures. We refer to this series of peaks, $C_{n}^{+}$, as the "multifragmentation" peaks since they arise predominantly from events in which there is a catastrophic disintegration of the $C_{60}$ molecule into many small fragments.

The manner in which energy is coupled into the $C_{60}$ system from the passage of a highly charged fast ion (of velocity, $v$ ) depends strongly on the impact parameter. The two principal distances of importance are the mean radius, $\bar{R},(3.55 \AA)$ of the $C_{60}$ "cage" on which are located the nuclei of the constituent carbon atoms, and the adiabatic distance, $b_{o}=\gamma \hbar v / E$ (=10 $\AA$ for $E=20 \mathrm{eV}$ ), for the excitation of the giant dipole plasmon resonance of energy $E$. This collective excitation of the 240 valence electrons of the $C_{60}$ molecule has been predicted $5,6,7$ and measured $8,9,10$ to have an energy of $20 \mathrm{eV}$ and a FWHM of about $10 \mathrm{eV}$. As an interesting aside demonstrating the ubiquity of collective resonances at different scales, we show in Fig. 3 a 
comparison of the giant dipole resonances observed in the $C_{60}$ molecule ${ }^{9}$ and in the ${ }^{12} \mathrm{C}$ nucleus ${ }^{11}$. Note that the excitation-energy scales differ by a factor of approximately $10^{6}$.

We have developed a quasi-classical model ${ }^{12}$ for the interaction between projectile and buckyball that gives the total excitation and single-plasmon excitation probabilities as a function of impact parameter. Preliminary to estimating the total interaction cross section, we consider the excitation of the giant dipole plasmon resonance by the Coulomb field of the xenon projectile. The effective number of plasmon excitations at an impact parameter $b$ is

$$
N(b)=\int d E \frac{f(E)}{E} \frac{2 Z_{P}^{2} e^{4}}{m v^{2}} \frac{1}{b^{2}}\left(\xi^{2} K_{1}^{2}(\xi)+\frac{1}{\gamma^{2}} \xi^{2} K_{0}^{2}(\xi)\right),
$$

where $\xi=E b / \gamma \hbar v, f(E)$ is the oscillator-strength distribution, and $Z_{P}$ is the charge state of the xenon ion. This expression, obtained in first-order perturbation theory, is consistent with that for the average energy transfer to a harmonic oscillator. ${ }^{13}$ The predicted ${ }^{5}$ oscillator strength is about 70 . We have therefore parameterized the oscillator-strength distribution $f(E)$ as a Gaussian, normalized to reproduce this and other known parameters of the resonance.

The excitation number $N(b)$ is large for $b \lesssim \bar{R}$. The strength of the plasmon resonance, combined with the high charge state of the xenon ion implies also that multiple excitations play an important role even at distances as big as the adiabatic distance, $b_{0}$. To make a realistic estimate of cross sections, we describe the plasmon excitations in terms of a "coherent state".14 The multi-plasmon excitation probabilities are then given by a Poisson distribution generated by $N(b)$. In particular, the probability for a one-plasmon excitation is $N(b) \exp (-N(b))$, and the total excitation probability is $1-\exp (-N(b))$. These two probabilities are illustrated in Fig. 4(a).

The total excitation probability reaches unity at an impact parameter of about $7 \AA$, still far outside the radius $\bar{R}$. To determine the total interaction cross section, it is therefore not necessary to consider explicitly reactions at the smaller impact parameters where the xenon ion may interact with individual electrons, and we can simply write this cross section as

$$
\sigma_{e x c}=2 \pi \int_{0}^{\infty} d b b(1-\exp (-N(b))) .
$$

The single-plasmon excitation cross section is

$$
\sigma_{1 p l}=2 \pi \int_{0}^{\infty} d b b N(b) \exp (-N(b))
$$

This estimate is reasonable since all the cross section comes from impact parameters much larger than $\bar{R}$ [c.f. Fig 4(a)]. The total interaction cross section obtained from the calculated values shown in Fig. 4(a) is $811 \AA^{2}$, whereas the single-plasmon cross section is $387 \AA^{2}$, i.e. $48 \%$ of the total. 
Our model should be valid for single-plasmon excitation involving large impact parameters where the linear-response and dipole approximations hold. It can be expected to break down at smaller impact parameters where multi-plasmon excitation occurs leading to multiple ionization, pair emission, and (at still smaller impact parameters) multifragmentation. The dominant decay mode of the single-plasmon excitation is thought to be via single-electron emission. 9,15 We therefore compare the calculated single-plasmon cross section (Eq. 3) to our measured $C_{60}^{+}$yield. The dependence on beam energy is illustrated in Fig. 4(b) for the projectile charge state $Z_{P}=18$. The weak dependence on beam energy is reproduced by the calculation. We were unable to determine accurate experimental cross sections. However rough estimates based on the calculated vapor pressure, the integrated beam current, and taking into account the experimental geometry, detector efficiency, etc, agree with the calculated values within a factor of about two. The slope of the calculated curve is insensitive to small variations in the total oscillator strength. We also considered the role of electron capture by the $\mathrm{Xe}$ ions as a production mechanism for $C_{60}^{+}$ions but at the high velocities (11-14 a.u.) of our beams, the charge-capture cross sections 16 are negligible compared to the cross sections for plasmon excitation.

At impact parameters less than about $7 \AA$ where the energy deposition becomes large, essentially all projectile/target interactions will result in multifragmentation. We have constructed a bond-percolation model ${ }^{12}$ to describe these fragmentation processes. $C_{60}$ is represented as a collection of lattice sites located at the positions of the carbon atoms. Each site is connected to its three nearest neighbors via bonds. We assume that each xenon ion deposits excitation energy in proportion to its pathlength through the hollow fullerene structure. The energy is then rapidly distributed in a uniform manner over the whole $C_{60}$-cluster. This leads to the breaking of individual bonds with a probability proportional to the total energy deposition, which in turn, is dependent on the impact parameter. The fragment-mass distribution calculated using this model compares well with the measured fragment mass spectrum (Fig. 5).

Although the giant dipole resonance is known to decay overwhelmingly by singleelectron emission 9,15 there should exist a relatively small $\left(\sim 10^{-4}\right.$ to $\left.10^{-5}\right)$ photon-decay branch. We calculated that we ought to be able to observe this decay and so we set up a VUV diffractiongrating spectrometer at $90^{\circ}$ to the incident beam direction looking for photons in the $20-\mathrm{eV}$ range. For these measurements the maximum ion-beam current ("unswept") and charge state were employed. After getting the background radiation down to acceptable levels, we found that the VUV spectrum in the region of interest (from $\sim 400 \AA$ to $\sim 1300 \AA$ ) was dominated by photons emitted from (mostly) singly charged carbon ions (C II). We were therefore unable to identify photons from the giant resonance. A typical spectrum is shown in Fig. 6. The measured peaks in terms of the photon energies and relative intensities agree well with those determined in earlier work with gaseous discharges. 17 
The electron configurations involved in the observed VUV transitions shown in Fig. 6 are all in the valence shells of $C$ II. These transitions are known 17 to have short ( $<1 \mathrm{~ns}$.) lifetimes. In our measurements we measured the lifetimes of the transitions relative to the arrival time of the beam pulse at the target. All of them displayed these expected short lifetimes, but interestingly, some of them, eg. the $687-\AA$ and the unresolved $(806+809)-\AA$ transitions shown in Fig. 6, exhibit also significant long-lived components on the order of $100 \mathrm{~ns}$. These long-lived components presumably reflect the production lifetimes for these particular configurations of $\mathrm{C} \mathrm{II}$ in the interaction of a xenon ion with $C_{60}$. We are currently trying to understand in more detail how these configurations arise and to see if they are connected in any direct way with the original bonding in the target molecule.

This work was supported by the U.S. Department of Energy, Office of Basic Energy Sciences, Contract W-31-109-ENG-38. We wish to thank B.J. Zabransky and C.A. Kurtz for their invaluable technical assistance.

* Present address: Dept. Physics and Astronomy, Univ. of Toledo, Toledo, $\mathrm{OH} 43615$ 


\section{References}

1. H.W. Kroto, J.R. Heath, S.C. O'Brien, R.F. Curl, and R.E. Smalley, Nature, $\underline{318}, 162$ (1985).

2. W. Krätschmer, L.D. Lamb, K. Fostiropoulos, and D.R. Huffman, Nature, 347, 354 (1990).

3. For reviews see, for example, E.E.B. Campbell et al, in Nuclear Physics Concepts in Atomic Cluster Physics, (Springer, Berlin, 1992) and in Proc. XVIII Int. Conf. on the Physics of Electronic and Atomic Collisions, Aarhus, July 21, 1993.

4. B. Walch, C.L. Cocke, R. Voelpel, and E. Salzborn, Phys. Rev. Lett. 72, 1439 (1994).

5. G.F. Bertsch, A. Bulgac, D. Tomanek, and Y. Wang, Phys. Rev. Lett. 67, 2690 (1991).

6. G. Barton and C. Eberlein, J. Chem. Phys. 95, 1512 (1991).

7. D. Östling, P. Apell, and A. Rosén, Europhys. Lett. 21, 539 (1993).

8. R.K. Yoo, B. Ruscic, and J. Berkowitz, J. Chem. Phys. 96, 911 (1992).

9. I.V. Hertel, H. Steger, J. deVries, B. Weisser, C. Menzel, B. Kamke, and W. Kamke, Phys. Rev. Lett. 68,784 (1992).

10. J.W. Keller and M.A. Coplan, Chem. Phys. Lett. 193, 89 (1992).

11. R.G. Allas, SS. Hanna, L. Meyer-Schützmeister, and R.E. Segel, Nucl. Phys. $\underline{58}, 122$ (1964).

12. T. LeBrun, H.G. Berry, S. Cheng, R.W. Dunford, H. Esbensen, D.S. Gemmell, and E.P. Kanter, Phys. Rev. Lett. 72 , 3965 (1994).

13. J.D. Jackson, Classical Electrodynamics (John Wiley and Sons, New York, 1962).

14. See, for example, E. Merzbacher, Quantum Mechanics (John Wiley and Sons, New York, 1970).

15. T. Drewello, W. Krätschmer, M. Fieber-Erdman, and A. Ding, Int. J. Mass Spectrosc. and Ion Proc. 124, R1 (1993).

16. A.S. Schlachter, J.W. Stearns, W.G. Graham, K.H. Berkner, R.V. Pyle, and J.A. Tanis, Phys. Rev. A 27, 3372 (1983).

17. R.L. Kelly and L.J. Palumbo, Atomic and Ionic Emission Lines below 2000 Angstroms, (NRL Report 7599. U.S. Govt. Printing Office, Washington DC, 1973). 


\section{Figure captions}

1. Schematic diagram of experimental arrangement.

2. Time-of-flight spectrum for positive fragments arising from bombardment of $C_{60}$ by $625-\mathrm{MeV} 136 \mathrm{Xe}^{35+}$ ions. The numbers given above some of the peaks are the ratios $(\mathrm{M} / \mathrm{Q})$ of fragment mass (amu) to charge.

3. Comparison of the giant dipole plasmon resonances observed in a) the $C_{60}$ molecule via photionization with synchrotron radiation 9 , and b) the ${ }^{12} C$ nucleus via the ${ }^{11} B(p, \gamma)^{12} C$ reaction. ${ }^{11}$

4. a) Calculated probabilities for the total interaction and for single-plasmon excitation as functions of the impact parameter, $b$, for $625-\mathrm{MeV}{ }^{136} \mathrm{Xe}^{35+}$ ions.

b) Calculated single-plasmon cross section (shown as a line) compared to the measured yields of $C_{60}^{+}$from $C_{60}$ bombarded by ${ }^{136} \mathrm{Xe}^{18+}$ ions in the energy range $420-625 \mathrm{MeV}$.

5. Measured mass distribution (solid points) for positive fragments arising from $C_{60}$ bombarded by $625-\mathrm{MeV}{ }^{136} \mathrm{Xe}^{35+}$ ions. The histogram is the distribution calculated on the basis of a multifragmentation model.

6. VUV photon spectrum measured with a position-sensitive detector at the focal plane of a grating spectrometer located perpendicular to the incident-beam direction. The xenon projectile energy was $625 \mathrm{MeV}$ and the average charge state (after foil stripping just upstream of the $C_{60}$ target) was $40+$. The peaks are labelled with identifications derived from the tabulations given in Ref. 17. 


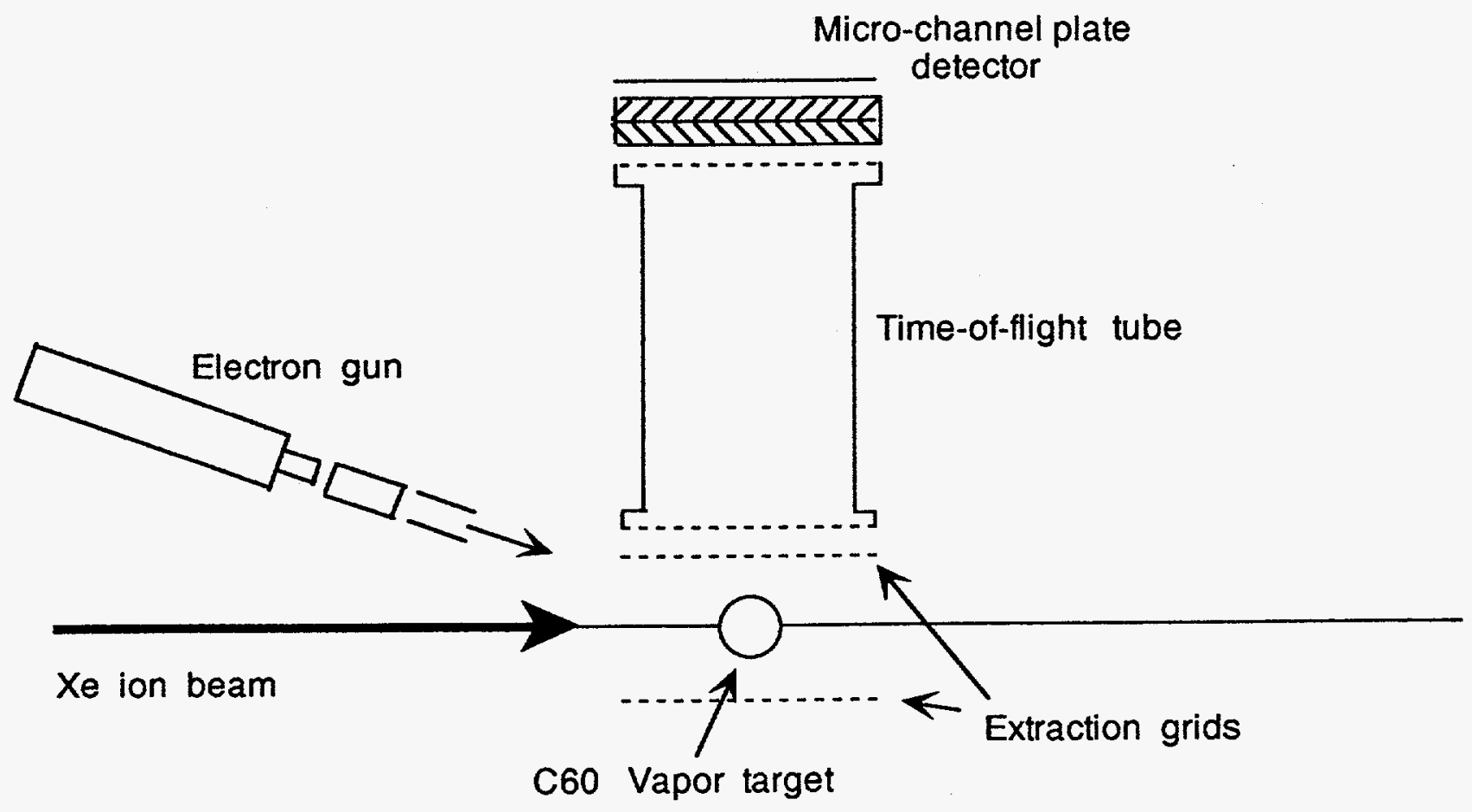

Fig. 1 


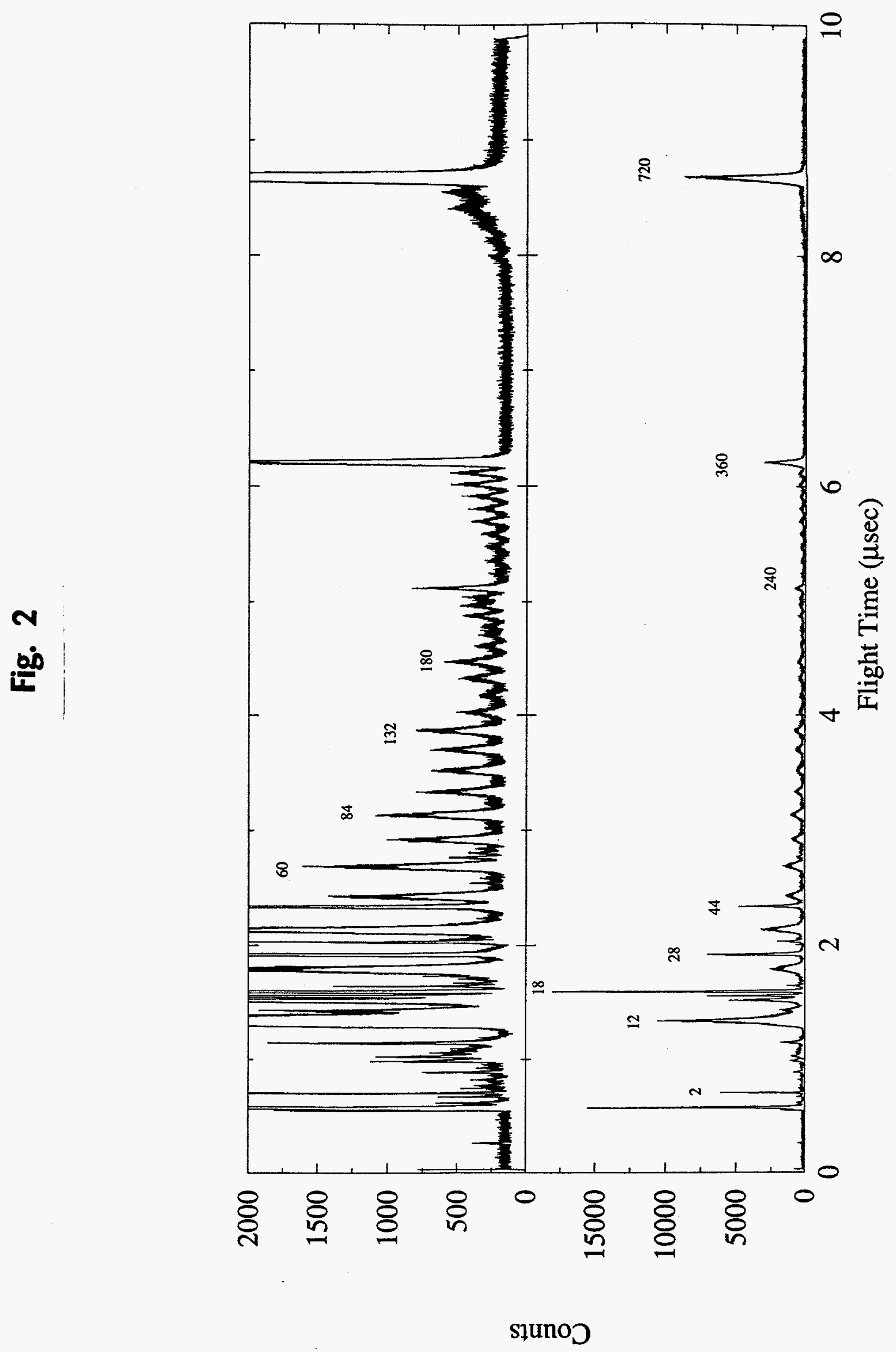



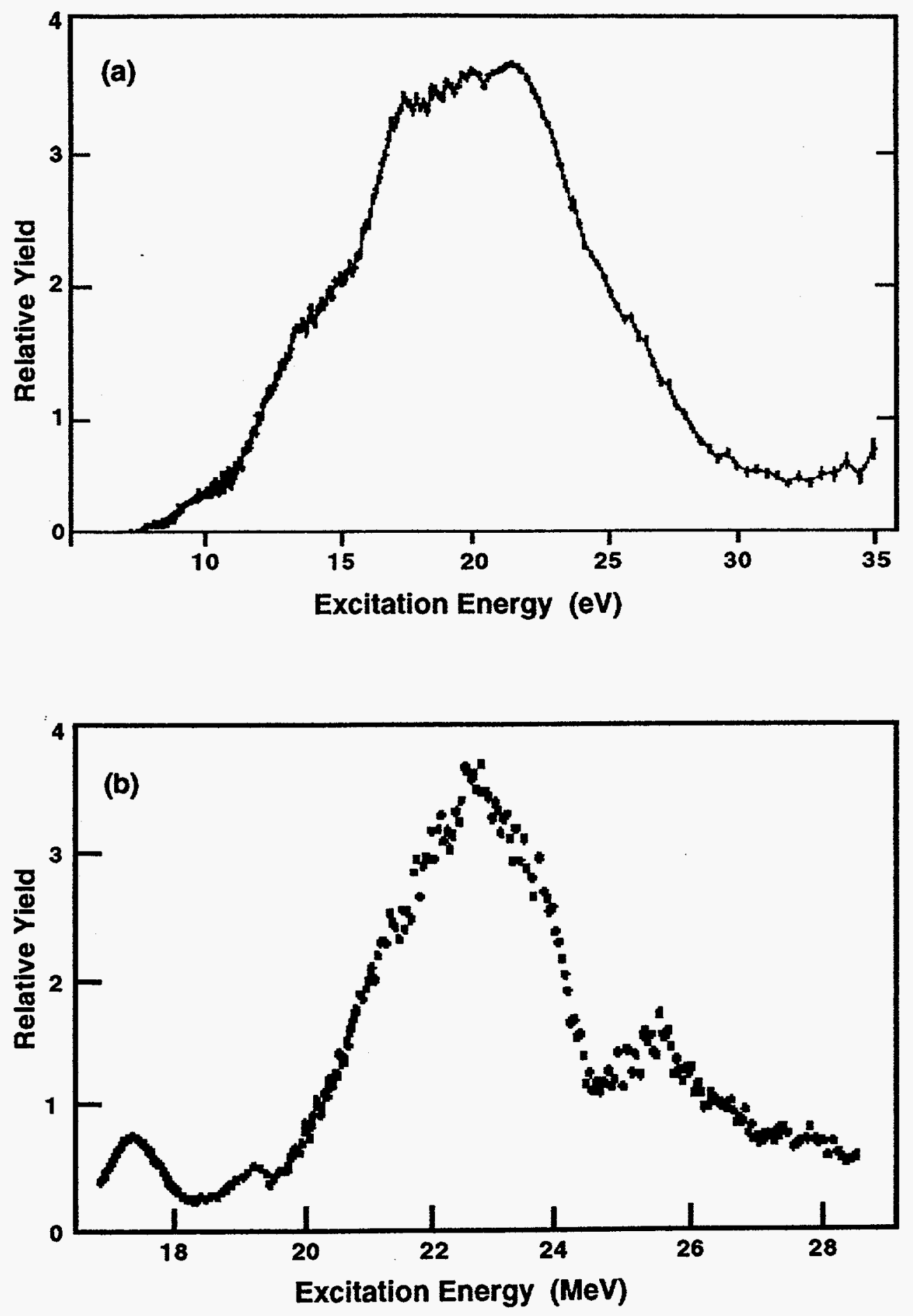

Fig. 3 

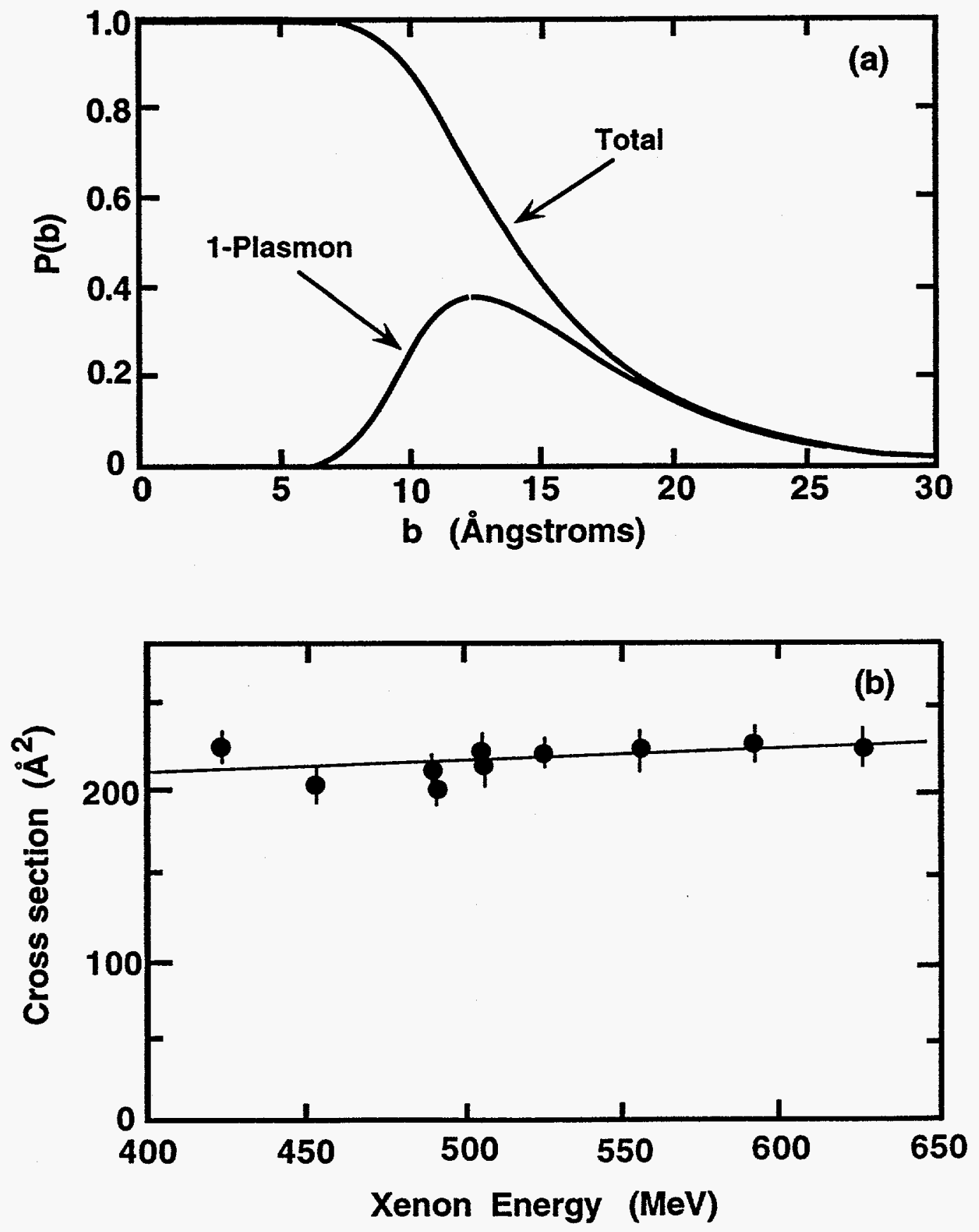

Fig. 4 


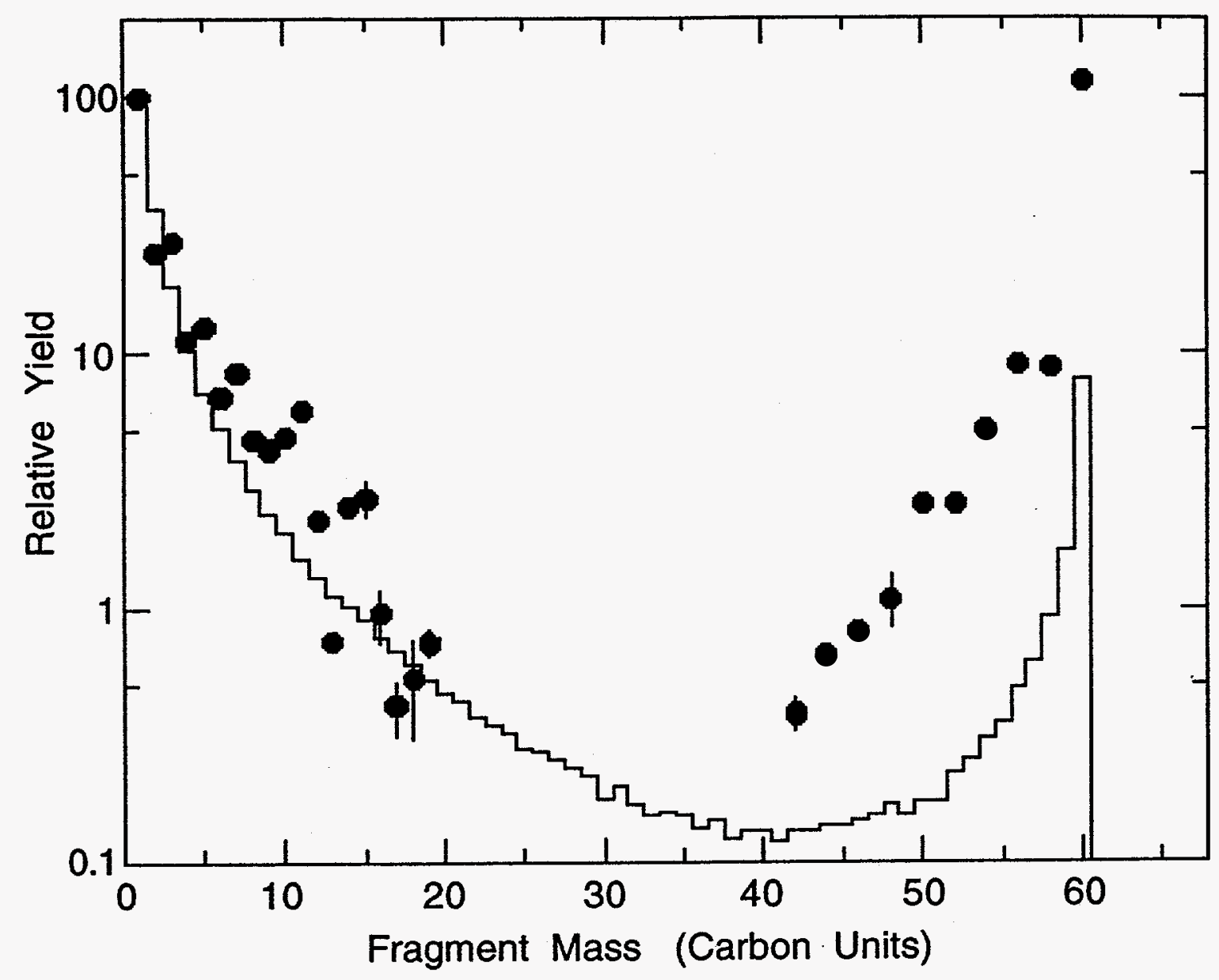

Fig. 5 


\section{DISCLAIMER}

This report was prepared as an account of work sponsored by an agency of the United States Government. Neither the United States Government nor any agency thereof, nor any of their employees, makes any warranty, express or implied, or assumes any legal liability or responsibility for the accuracy, completeness, or usefulness of any information, apparatus, product, or process disclosed, or represents that its use would not infringe privately owned rights. Reference herein to any specific commercial product, process, or service by trade name, trademark, manufacturer, or otherwise does not necessarily constitute or imply its endorsement, recommendation, or favoring by the United States Government or any agency thereof. The views and opinions of authors expressed herein do not necessarily state or reflect those of the United States Government or any agency thereof.

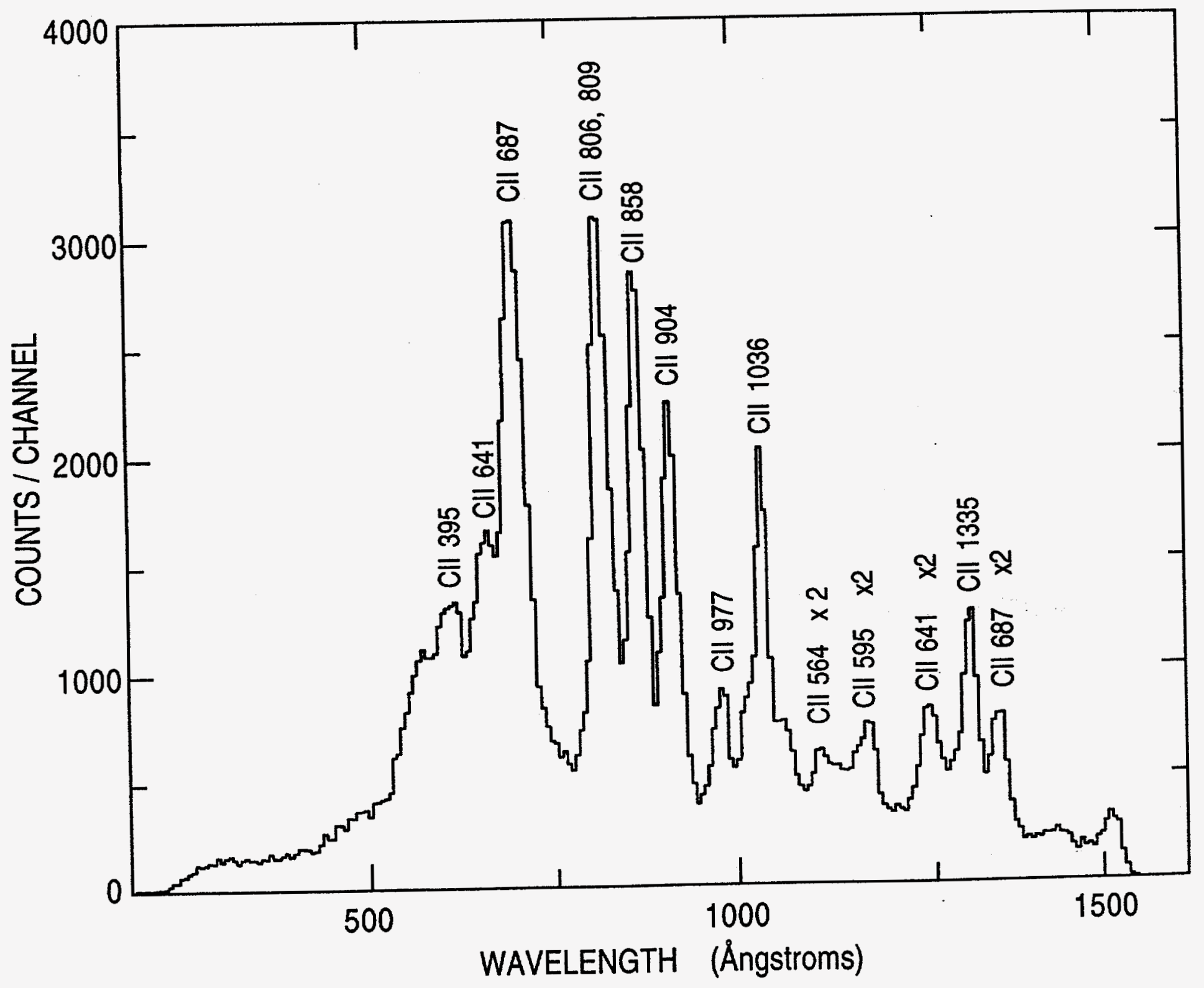

Fig. 6 